\title{
Traduire
}

Revue française de la traduction

$218 \mid 2008$

De traduction en retraduction

\section{Traduire Machiavel}

\section{Thierry Ménissier}

\section{(2) OpenEdition \\ Journals}

Édition électronique

URL : http://journals.openedition.org/traduire/895

DOI : 10.4000/traduire.895

ISSN : 2272-9992

Éditeur

Société française des traducteurs

Édition imprimée

Date de publication : 15 juin 2008

Pagination : 29-42

ISSN : 0395-773X

\section{Référence électronique}

Thierry Ménissier, «Traduire Machiavel », Traduire [En ligne], 218| 2008, mis en ligne le 15 juin 2008, consulté le 01 mai 2019. URL : http://journals.openedition.org/traduire/895 ; DOI : 10.4000/ traduire.895 


\title{
Traduire Machiavel
}

\author{
THIERRY MÉNISSIER
}

"Quant aux mensonges des habitants de Carpi, je peux leur en revendre à tous, car il y a beau temps que je suis devenu docteur en ce domaine, au point que je ne voudrais pas de Francesco Martelli pour commis. Depuis longtemps, en effet, je ne dis pas ce que je pense, ni ne pense ce que je dis, et, si je dis parfois la vérité, je la cache parmi tant de mensonges quil est difficile de la découvrir »(1).

L'œuvre de Machiavel (1469-1527) se trouve souvent précédée par la réputation de l'auteur, et plus souvent encore interprétée ou déformée à la lumière du terme en "isme " dérivé de son nom : la réaction du lecteur au machiavélisme (à savoir le tour d'esprit de celui qui voulant la fin, se moque de la nature et surtout de la qualité morale des moyens) tend à se substituer à la lecture attentive des textes originaux, même chez un public savant. Non que Machiavel, dans ses ouvrages, ne se montre pas machiavélique, au contraire, de nombreux passages de l'œuvre incitent le lecteur à adopter le point de vue machiavélique, et cela jusqu'au blasphème(2). Mieux encore, le fameux précepte énoncé dans le chapitre $\mathrm{XV}$ du Prince, celui de s'en tenir à la verità effettuale

(1) Lettre de Machiavel à Francesco Guicciardini, 17 mai 1521, traduction Christian Bec dans Machiavel : 1996, p. 1254-1255.

(2) Voir comme exemple d'un passage à la tonalité machiavélique et tendant au blasphème : "...Je crois que cela provient de la cruauté bien ou mal employée. On peut nommer " bien employées " (si du mal il est permis de dire du bien) les cruautés qui se font tout d'un coup, en vertu de la nécessité de sécurité, et sur lesquelles on n'insiste pas après coup, mais que l'on convertit pour le plus grand profit possible de ses sujets. "Mal employées ", ce sont celles qui, même rares au début, croissent avec le temps au lieu de s'éteindre. Ceux qui se comportent de la première manière peuvent, avec l'aide de Dieu et des hommes, avoir quelques remèdes à leur disposition... " (Le Prince, chapitre VIII, dans Machiavel, 2007). 
della cosa, "la vérité des faits et des effets de la chose [politique] ", dont on sait qu'il conditionne aussi bien l'écriture machiavélienne de la politique que la description de ce que l'auteur estime être la meilleure forme de psychologie pour l'homme d'action, accouche du tour d'esprit machiavélique :

Beaucoup se sont imaginé des républiques et des principautés que jamais on n'a véritablement ni vues ni connues, car il y a un tel écart entre la façon dont on vit et celle dont on devrait vivre, que celui qui délaisse ce qui se fait pour ce qui devrait se faire apprend plutôt à se perdre qu'à se sauver. En effet, l'homme qui en toutes choses veut faire profession de bonté se ruine inéluctablement parmi tant d'hommes qui n'ont aucune bonté. De là il est nécessaire à un prince, s'il veut se maintenir au pouvoir, d'apprendre à pouvoir n'être pas bon, et d'en user et n'en pas user selon la nécessité(3).

Pourtant il est aisé de montrer qu'en dépit de sa réputation bien méritée, l'œuvre ne se laisse pas réduire à une seule interprétation, et qu'elle recèle au contraire des significations multiples : notamment, l'auteur républicain favorable à la liberté des peuples opposés à toute domination arbitraire vient contrebalancer la réputation de machiavélisme quand bien même s'agit-il pour lui de théoriser les conditions d'une "république en armes" (repubblica armata). Dans les pages qui suivent je voudrais brièvement et à partir de mon expérience personnelle donner une idée de ce que signifie la traduction d'un tel auteur - à vrai dire, la traduction d'un auteur exceptionnel tant par son style (il pratique un italien fait de fulgurante sobriété), que par le caractère incroyablement pénétrant de ses vues et par sa très grande postérité dans la tradition de pensée politique occidentale. Mais j'invite le lecteur de ce témoignage à prendre au sérieux l'avis qu'il rendait sur lui-même dans sa correspondance privée à son ami Guichardin (voir l'exergue de cet article), et à le considérer comme un salutaire avertissement pour chacun de ses traducteurs : malgré la tendance qu'avait Machiavel à s'affubler de défauts qu'il n'avait pas, comment traduire les œuvres d'un homme qui confie ne jamais dire ce qu'il pense ni ne jamais penser ce qu'il dit?

(3) Ibidem, chapitre XV, p. 75. 


\section{Les origines de la langue de Machiavel : la rencontre d'idiomes variés dans une expérience unique}

Il y a quelques années, j'ai rédigé pour une collection universitaire un " vocabulaire de Machiavel ", dans lequel je me suis moins penché sur la langue de départ de l'auteur que sur la possibilité de traduire intellectuellement les notions dont il se sert dans un lexique de philosophie politique usuel(4). C'est cependant à cette occasion que j'ai achevé de prendre conscience du caractère profondément étonnant de la langue dans laquelle s'exprime Machiavel ; il compose une langue originale à partir de traditions linguistiques variées n'ayant que peu de rapport les unes avec les autres, mais qui se sont en quelque sorte rencontrées dans la carrière de l'auteur, haut fonctionnaire de la République florentine, dite du Grand Conseil (Consiglio maggiore), de 1498 à 1512. Une fois limogé de son poste par le retour au pouvoir des Médicis, à l'issue d'une crise qui a désarçonné acteurs et observateurs et démontré le caractère caduc des matrices traditionnelles d'intelligence du fait politique, Machiavel écrit à son ami Francesco Vettori que pendant les quatorze années passées aux affaires, il a appris "l'arte dello stato »(5), ce qu'on pourrait traduire par «le métier public ». Cette affirmation me suggère deux remarques.

Elle me permet d'abord de rappeler un fait bien connu, qui permet d'évaluer la difficulté du traducteur. Ce "métier» se trouve tout à fait lié à une pratique d'écriture - dans les différentes fonctions qu'il a occupées, Machiavel était astreint à dicter plusieurs dizaines de lettres par jour -, si bien que "l'arte dello stato " peut s'entendre également comme " la pratique de la langue administrative ". La variété de ses emplois a permis à l'auteur de fréquenter et d'employer plusieurs idiomes techniques différents tous inscrits dans la langue toscane, qui de son côté devait tant à l'expression des marchands florentins(6) : l'idiome des diplomates obligés à la relation des affaires de la grande

(4) Ménissier : 2002.

(5) Lettre de Machiavel à Francesco Vettori, 10 décembre 1513, dans Machiavel, Euvres, 1996 p. 1240.

(6) Cf. Bec : 1967. 
politique, celui des hauts fonctionnaires devant composer des rapports techniques, celui des historiens en langue vulgaire de l'humanisme civil du Quattrocento, attachés à célébrer la supériorité du régime républicain sur ses rivaux milanais ou pontifical, et tout simplement aussi celui des artisans, puisqu'il fournit un matériau de premier ordre à un auteur dont la visée est avant tout pratique et jamais purement théorique. Tout cela est bien connu des spécialistes de l'étude de la langue de Machiavel(7). J'ajouterai pour ma part une dimension, importante quoique rarement interprétée au niveau où elle devrait l'être: dramaturge qui connut un certain succès, Machiavel ne se coupe jamais de la tradition théâtrale, surtout pas en ce qui concerne sa langue construite à partir de la notion de personnage, sinon en fonction de la matrice dialogique qui lui est implicitement liée - j'ai insisté ailleurs sur ces deux aspects, notamment sur " le système des personnages" (son œuvre en comprend plus de 1500 ) et sur la nécessité d'une approche méthodologique distinguant les "dialogues avec des amis" (les Discours sur la première décade de Tite-Live me paraissent relever de ce cas de figure) des "dialogues avec des adversaires" (tels Le Prince et les Histoires florentines)(8). On pourrait même ajouter que le genre de théâtre qui a influencé le Secrétaire, c'est avant tout la beffa florentine, cette farce méchante destinée à une mise à mort symbolique d'un adversaire dans la joute sociale(9). La forme, mais aussi le contenu même de l'œuvre en sont affectés, puisque la recommandation d'user de la ruse entretient des liens subtils avec l'inganno, la tromperie véritable ressort de la beffa. Qu'on apprécie à cette lumière la sentence que voici : "les hommes sont si naïfs, et ils obéissent tellement aux nécessités présentes, que celui qui trompe trouvera toujours quelqu'un qui se laissera tromper $"(10)$.

(7) Trois études récentes en français permettent d'approfondir la question proprement linguistique dans l'œuvre de Machiavel : Fournel, in Fumaroli (dir.) : 1999 ; Fournel et Zancarini : 2000 ; Gerbier, in Gaille-Nikodimov et Ménissier : 2006.

(8) Voir Ménissier : 2001a.

(9) Cf. Celse : 1972 et Marietti : 1972.

(10) «Sono tanto semplici gli uomini, e tanto ubbidiscono alle necessità presenti, che colui che inganna troverrà sempre chi si lascerrà ingannare ", Le Prince, chapitre XVIII, ma traduction p. 85. 
Forgée sous la contrainte des temps, liée à la crise profonde issue de la nouvelle donne de la politique italienne et internationale à partir de 1494, la langue machiavélienne ne consiste pas pour autant en un patchwork d'idiomes assemblés dans l'urgence. Véritablement personnelle, inimitable et fascinante - "sèche et subtile comme l'air de Florence ", comme a écrit Nietzsche - elle reflète l'acuité d'une pensée de la politique qui se confond avec un style. Une fois limogé et exilé dans sa ferme de Sant'Andrea in Percussina, à quelques dizaines de kilomètres de Florence, le Secrétaire réfléchit sur son expérience et médite les classiques latins de la pensée politique (entre lesquels TiteLive, Tacite, César et, parmi les Grecs, lus en traduction, Xénophon) ; c'est à cette occasion, loin du pouvoir et totalement frustré, qu'il écrit ses œuvres majeures. Or, on s'accorde à reconnaître l'importance décisive de cette retraite forcée sur la constitution de la langue qui a fait la réputation de l'auteur. Plusieurs faits attestent d'ailleurs que l'auteur a eu conscience de l'effort nécessaire pour l'unifier, et il l'a sans cesse polie, au point que, dans de véritables exercices, il a entrepris de réécrire plusieurs fois les mêmes événements politiques ou épisodes historiques : conçue dynamiquement, telle une œuvre musicale vivante, elle comprend donc des motifs fondamentaux sans cesse repris et réinterprétés.

\section{L'invention de la philosophie politique moderne à partir de la langue machiavélienne}

Il faut d'autre part souligner l'importance du dessein machiavélien de composition d'une langue qu'il veut, selon l'impératif de la verità effettuale della cosa, la plus adaptée possible à la nouvelle donne de la politique. Ce faisant, le Secrétaire florentin contribue à forger des notions, ou mieux encore un style de conceptualisation du pouvoir, qui se confond avec la naissance de la philosophie politique moderne. En ce sens, tous les penseurs modernes de la politique sont les héritiers de Machiavel. Ici la réflexion sur la langue est également décisive : je vais envisager un seul cas de figure, mais cardinal, celui qui concerne la 
constitution de véritables synthèses conceptuelles, ainsi qu'on le voit premièrement avec les termes principato et repubblica, et deuxièmement avec le terme stato.

Le premier chapitre du Prince débute par cette déclaration :

Tous les États, toutes les puissances qui ont eu et ont pouvoir sur les hommes ont été et sont ou bien des républiques ou bien des principautés [Tutti gli stati tutti e dominii che hanno avuto et hanno imperio sopra gli uomini, sono stati e sono o republiche o principati].

Cette déclaration contient en germe l'intégralité du programme que Machiavel s'est fixé : Le Prince théorise les usages typiques des "principautés ", tandis que les Discours sur la première décade de Tite-Live (vraisemblablement composés entre 1513 et 1518) et les Histoires florentines (1525) examinent la vie des républiques. Or, si cette formulation lapidaire doit retenir l'attention, c'est que les deux formes ultimes d'institutions politiques identifiées par le Florentin sur le ton de l'évidence, "principautés " et "républiques " n’ont aucune existence empirique! Les formes d'institutions sont multiples, chacun le sait, à commencer par Machiavel dont toute la carrière s'est déroulée au sein des divers duchés (par exemple Milan), monarchies (France), républiques constitutionnelles dont la base électorale était plus ou moins large (Florence sous ses différents régimes), oligarchie (Venise), etc. L'enjeu recouvert par un mot tel que "principato " doit être recherché ailleurs, c'est-à-dire dans un autre ordre de faits : il se distingue notoirement de "monarchia ", lequel désigne le pouvoir féodal, conçu par le Florentin dans toute sa complexité (soit en apparence un pouvoir personnalisé par le roi, mais qui s'appuie sur la tradition d'une dynastie, sur le prestige d'une maison, sur la puissance - notamment financière - d'un domaine, et sur la réalité d'un territoire)(11); et il vise à rendre compte de la situation d'un homme seul réussissant à prendre et à conserver le pouvoir grâce à une combinaison de chance et de talents.

(11) De retour de mission, Machiavel a rédigé plusieurs rapports sur l'organisation du pouvoir de la monarchie française, à commencer par un très intéressant Ritratto delle cose di Francia (Portrait des choses de France) daté de 1510. 
Autrement dit - et sur ce point se fait sentir l'étrange travail que Machiavel fait subir à sa langue - «principauté » est un terme voué à capter les différentes possibilités au sein desquelles évolue l'homme qui prend le pouvoir et qui entend le garder. Constitutionnel en apparence, le terme vise en réalité une phénoménologie de la prise du pouvoir, comprenant une psychologie, une stratégie, une théorie de la chance, sinon une métaphysique. De son côté, le terme "repubblica", tel qu'on le voit à l'œuvre dans les Discours, embrasse toutes les formes institutionnelles dans lesquelles le pouvoir est partagé d'une manière ou d'une autre. Aussi, une monarchie constitutionnelle telle que l'Angleterre en connaît une depuis le XVIIe siècle serait-elle décrite en termes machiavéliens comme une... république !

On peut se livrer à une analyse comparable à propos d'un terme encore plus important pour la postérité intellectuelle de l'Europe, le terme "stato » : son usage n'est pas fixé au moment où Machiavel entreprend de lui donner une signification précise pour la pensée politique(12). Tandis qu'il désigne littéralement "l'établissement " de quelqu'un particulièrement, le patrimoine privé comme dans l'expression "lo stato dei Medici ", qu'on pourrait rendre par " la maison Médicis" -, le Florentin met l'accent sur la nature du pouvoir permettant de fonder une autorité de type public. Pour le traducteur, la difficulté consiste à prendre acte de la diversité initiale du terme - diversité qui rend compte aussi bien du foisonnement de la langue que de l'acuité des Toscans en matière de situations sociales -, tout en comprenant la tâche auquel s'astreint l'auteur, mais sans pour autant " écraser " ou "saturer " la signification du texte par le mot d' « État " entendu de manière contemporaine(13). Il s'agit donc de restituer le travail double de reconstitution synthétique et de définition analytique auquel donne lieu le terme "stato", en évitant de donner ce dernier à lire à travers l'épaisseur asphyxiante de l'histoire du mot "État ». Ce problème

(12) Voir Rubinstein : 1980-1981 et Tenenti : 1987.

(13) J'ai examiné ce problème dans une étude centrée sur le chapitre I du Prince, cf. Ménissier : $2001 \mathrm{~b}$. 
m'inspire deux remarques : d'une part, il est nécessaire d'établir sans cesse une tension entre la langue de départ et la langue d'arrivée, et une heureuse traduction de Machiavel se reconnaît, me semble-t-il, à sa capacité à ne jamais se dérober à la tension : ni radicalement " passéiste" (l'écueil le plus redoutable se situe sans doute dans la tentation, souvent présente chez les traducteurs savants, qui consiste à faire de lui un auteur enfermé dans son époque), ni brutalement " contemporanéiste" (il faut accepter le fait que, quand bien même Machiavel " nous parle ", il n'est pas directement notre contemporain), elle doit révéler sans lourdeur tant l'intelligence que le Florentin avait des situations qu'il décrit que son inventivité dans les notions qu'il soumet à l'analyse. D'autre part, et corrélativement, si l'on veut saisir le travail intellectuel qui est en jeu, il faut sans doute adopter le point de vue inverse du rapport traditionnellement établi entre la langue et la pensée : n'en déplaise aux philosophes désireux de reconnaître l'un des leurs en la figure du Florentin, au lieu que la première soit regardée comme la servante de la seconde, le travail qui s'opère grâce à celle-ci n'a pour dessein que de préciser les significations de celle-là. Le travail de la pensée vise à préciser les termes de base d'une langue vouée à la lucidité la plus grande possible en un temps où les matrices traditionnelles se sont trouvées désavouées.

\section{Machiavel et la France}

Afin de donner à ce rapide parcours un relief supplémentaire, j'ajouterai une remarque, qui consiste en réalité en une piste de réflexion à poursuivre. Il faudrait un jour examiner en détail la présence de Machiavel dans notre histoire nationale par le biais des traductions régulières de ses œuvres, en prêtant attention au contexte politique dans lequel ces dernières prennent sens, d'une part, et, de l'autre, en scrutant la manière dont la pensée machiavélienne a sous cette forme plus ou moins infléchi ce contexte. Machiavel apparaît presque comme un auteur, sinon comme un acteur de l'histoire de France, tant ses apparitions dans notre langue semblent pleines de 
sens, et tant elles donnent à réfléchir à propos de la diffusion des idées et du type d'influence des lettres sur l'action des politiciens. Je n'en donnerai ici que trois exemples.

Premièrement, dans les années 1570 , sa présence à la cour des Valois, et plus particulièrement dans ce qu'on nommait avec détestation « le parti des Italiens " autour de Catherine de Médicis, a incontestablement joué un rôle dans la politique menée durant les plus dures années des guerres de religion. On peut à cet égard mentionner la traduction en 1571 du Prince et des Discours par Jacques Gohory (15201576). Si la « responsabilité » du Secrétaire dans le massacre de la saint Barthélemy de l'été 1572 n'est que très indirecte, voire franchement improbable (Machiavel ne recommande nulle part le massacre de masse, une telle exaction détonne même dans l'économie de la violence qu'il prône), sa réputation exécrable a contribué à la mise en place du puissant courant intellectuel connu sous le nom d'antimachiavélisme, dont la vigueur théorique a conféré à la monarchie française les éléments de l'art moderne de gouverner. La notion cardinale de souveraineté a notamment été inventée en partie en réaction à la guerre civile qui faisait rage dans le royaume. On peut consulter sur ce point deux ouvrages très importants de la fin du XVIe siècle : L'Antimachiavel d'Innocent Gentillet et les Six livres de la République de Jean Bodin, l'un et l'autre parus en 1576.

Deuxième exemple, entre le siècle du classicisme et celui des Lumières, les traductions et commentaires de Nicolas Amelot de la Houssaye (1634-1706, traducteur du Prince en 1684) ont contribué à transformer la réception de Machiavel en France, en faisant basculer son image d'auteur de la raison d'État (qui est son deuxième avatar français après celui de sombre conseiller des tyrans, précisément hérité de l'antimachiavélisme du XVIe siècle) vers celle d'un auteur républicain, soucieux d'instruire les peuples afin de les libérer de la tutelle des rois (ce qui est la lecture de philosophes tels que Spinoza et Rousseau). Il écrivait ainsi dans la préface de sa traduction : "Au reste, je dirai, que Machiavel, qu'on fait passer partout pour un Maître de tyrannie, l'a détestée plus que pas un homme de son temps". 
Dernier exemple, mais non le moindre, Charles-Philippe-Toussaint Guiraudet (1754-1804), qui avait été député du Gard à la Constituante et Secrétaire général au Secrétariat aux relations extérieures, mort préfet de la Côte d'Or, fut l'auteur en 1799 d'une traduction complète de l'œuvre machiavélienne en neuf volumes, dans laquelle le terme "mutazioni", si fréquent chez un Machiavel penseur des bouleversements historiques (sinon apôtre du renouvellement périodique du pouvoir), est rendu significativement par... "révolution ". Tout s'est sans doute passé alors comme si la république issue de la Révolution, peinant à trouver un second souffle, avait cherché un " prince nouveau ", sorte de nouveau César (Borgia ?), Bonaparte devenant rapidement Napoléon.

Les traductions de Machiavel accompagnent et stimulent notre histoire politique nationale : toute apparition du Florentin dans notre paysage hexagonal signifie quelque chose et sans doute y ajoute son dynamisme créateur. C'est dans cette optique qu'il convient de resituer et d'envisager les nombreuses traductions dont il fait l'objet depuis 1990, dans le contexte de l'érosion de la pensée marxiste et du développement d'une idéologie de type libéral ; le Machiavel républicain qui revient sur le devant de la scène théorique, dans l'espoir de repolitiser l'espace public, fait paradoxalement pièce au machiavélisme stratégique et apolitique déployé par la pensée néolibérale.

\section{Conclusion : les «fortunes" de la virtì}

Je veux conclure cet article en mettant l'accent sur l'engagement qu'implique le fait de traduire Machiavel. Avec ce terme, je n'entends pas désigner l'acribie nécessaire afin de restituer la subtilité du Florentin, mais la résolution morale. En effet, un des problèmes majeurs dont hérite tout traducteur réside dans le choix à opérer à propos du mot de "virtù ", dont on pourrait dire qu'il constitue le cœur de la théorie morale de Machiavel. S’il est sous-déterminé dans l'italien du XVIe siècle - ce qui est lié à son origine latine, la virtus désignant d'une manière générale la force virile ou le courage - chacun 
sent que lui aussi se transforme sous la plume de l'auteur. Mais surgit une équivoque majeure : ou bien on le minore en le rendant par " talent", ce qui désigne la compétence politique envisagée d'un point de vue technique, ou bien on tient ferme sur l'aspect moral du terme en le traduisant par "vertu", et il faut en accepter les conséquences. Les deux options ne sont pas réconciliables, il y a même un monde entre les deux. Qu'on en juge avec le cas paradigmatique du chapitre VII du Prince, dont la traduction constitue une sorte de test de référence - pour confesser la vérité, le respect que je dois à Machiavel m'incline à penser qu'il s'agit plutôt d'un révélateur du tempérament du traducteur ou de sa capacité à supporter la pression morale imposée par le Florentin. Ou bien, en effet, on fait dire au texte que César Borgia a réussi à prendre le pouvoir en Romagne grâce à son (indéniable) talent, lequel présentait d'ailleurs de multiples facettes : audace, intelligence de la situation, courage physique, psychologie, réalisme dans le rapport des forces, absence de scrupules. Ou bien on assume le fait que la sanglante ou cruelle réussite de César est qualifiée par le Florentin d'acte vertueux, au sens moral du terme, et l'on accepte de se faire le passeur de monstruosités.

Chez Machiavel, la virulence du texte ne peut jamais s'éteindre sous le commentaire savant : il prône une conception du bien public qui redéfinit le rapport entre morale et politique. Ce qui est vertu en morale peut aisément devenir un vice pour l'homme politique ; par exemple la libéralité, à savoir la capacité à généreusement dépenser l'argent, tend à appauvrir les finances publiques. A contrario, ce qui est moralement vicieux peut non moins aisément se révéler une utile vertu ; par exemple la ladrerie, pour les raisons contraires du cas évoqué, permet de sauvegarder le Trésor ; et mieux ou pire encore : la rapacité, qui fait dépenser l'argent pris aux autres et passer chez soi pour libéral, peut être considérée comme une heureuse disposition en fonction de l'impératif suprême de "maintenir l'État " (mantenere lo stato) selon une expression récurrente qui donne le ton de l'éthique machiavélienne du pouvoir. Or, en conquérant la Romagne et en l'associant aux États pontificaux, César Borgia a agi vertueusement, puisque la fai- 
blesse des Italiens tenait à leur effective dispersion face à l'ennemi français ou espagnol, et que l'unité nationale constituait le bien suprême pour sauver l'Italie. Ce que nous apprend Machiavel, c'est que la valeur politique n'est jamais une affaire de morale, mais le plus souvent une question amorale ou même franchement immorale - tel est le scandale que doit assumer (et à mon sens, préserver comme une leçon irremplaçable) la postérité de ses traducteurs.

\section{Thierry.Menissier@upmf-grenoble.fr}

Thierry Ménissier, agrégé de philosophie et docteur de l'EHESS en études politiques, est maître de conférences à l'Université Pierre Mendès France - Grenoble 2. Principaux ouvrages publiés : Machiavel, la politique et l'histoire. Enjeux philosophiques (P.U.F., 2001); Machiavel, Le Prince ou le nouvel art politique (P.U.F., 2001, dir. en collaboration avec Y.C. Zarka) ; Lidée de contrat social. Genèse et crise d'un modèle philosophique (dir. en collaboration avec J.-P. Cléro, Ellipses, 2004); Éléments de philosophie politique (Ellipses, 2005); Lectures de Machiavel (dir. en collaboration avec M. Gaille, Ellipses, 2006); Lidée d'empire dans la pensée politique, historique, juridique et philosophique (dir., L'Harmattan, 2006).

\section{Références bibliographiques}

Bec, Christian, Les marchands écrivains. Affaires et humanisme à Florence, 1375-1434, Paris-La Haye, Mouton, 1967.

Celse, Mireille, "La beffa chez Machiavel, dramaturge et conteur ", in Ronchon André (dir.), Forme et signification de la beffa dans la littérature italienne de la Renaissance, Cahiers du C.I.R.R.I., Université de Paris III-Sorbonne Nouvelle, 1re série, $\mathrm{n}^{\circ}$ 1, 1972.

Fournel, Jean-Louis, " Rhétorique et langue vulgaire en Italie au XVIe siècle : la guerre, l'amour et les mots ", in Fumaroli Marc (dir.), Histoire de la rhétorique dans l'Europe moderne, 1450-1950, Paris, P.U.F., 1999, p. 313-339. 
Fournel, Jean-Louis et Zancarini, Jean-Claude, "Sur la langue du Prince : des mots pour comprendre et pour agir", dans leur traduction du Prince, accompagnée de l'édition du texte italien par G. Inglese, Paris, P.U.F., 2000, p. 545-610.

Gerbier, Laurent, « La composition de la langue civile. Sources, enjeux et construction de l'écriture politique machiavélienne ", in GailleNikodimov Marie et Ménissier Thierry, Lectures de Machiavel, Paris, Ellipses, 2006, p. 51-91.

Machiavel, Euvres, éditées et traduites par Christian Bec et Frédérique Verrier, Paris, Robert Laffont, 1996.

Machiavel, Le Prince, traduction Th. Ménissier, Paris, Hatier, collection «Les classiques de la philosophie ", 1999 ; nouvelle édition dans la collection « Philo et compagnie », 2007.

Marietti, Marina, "Aspects de la beffa dans les Istorie fiorentine de Machiavel ", in Ronchon André (dir.), Forme et signification de la beffa dans la littérature italienne de la Renaissance, Cahiers du C.I.R.R.I., Université de Paris III-Sorbonne Nouvelle, 1re série, $\mathrm{n}^{\circ}$ 1, 1972.

Ménissier, Thierry, Machiavel, la politique et l'histoire. Enjeux philosophiques, Paris, P.U.F., 2001a.

Ménissier, Thierry, "Les mots du pouvoir et les modes de la pensée politique ", in Zarka, Yves Charles et Ménissier Thierry (dir.), Machiavel. Le Prince ou le nouvel art politique, P.U.F., collection "Débats philosophiques ", 2001b, p. 13-46.

Ménissier, Thierry, Le vocabulaire de Machiavel, Paris, Ellipses, 2002.

Rubinstein, Nicolaï, "Notes on the word stato in Florence before Machiavelli ", in Per Federico Chabod, I, Lo stato e il potere nel Rinascimento, Annali della Facoltà di Scienze politiche, Università di Perugia, 1980-1981, Materiali di Storia, 5, pp. 137-146 ou in Florilegium Historiale: Essays Presented to Wallace Ferguson, Rowe, J.G \& Stockdale, W.H (eds), Toronto ; Toronto University Press, 1971, pp. 314-326. 
Tenenti, Alberto, "Archeologia medievale della parola Stato " et « La nozione di "stato" nell'Italia del Rinascimento ", in Stato : un'idea, una logica, Bologne, Il Mulino, 1987, pp. 15-51 et 53-97. 